\title{
HARMONIC WAVE PROPAGATION IN AN INFINITE ELASTIC MEDIUM WITH A PERIODIC ARRAY OF CYLINDRICAL PORES $\dagger$
}

\author{
T.-C. MA $\ddagger$, R. A. ScotT AND W. H. YANG \\ Department of Applied Mechanics and Engineering Science, University of Michigan, Ann Arbor, \\ Michigan 48109, U.S.A.
}

(Received 25 August 1979, and in revised form 5 February 1980)

\begin{abstract}
The propagation of plane harmonic waves in an elastic medium in which there is a periodic distribution of infinitely long, cylindrical pores is analyzed. The waves propagate perpendicular to the pores and the approach used is a finite element method based on Galerkin's technique. Results on dispersion, for several values of porosity, and on strain energy distributions within a typical half-cell are given. Also, as a check on the dynamic results, equivalent elastic moduli are derived by using a static analysis.
\end{abstract}

\section{INTRODUCTION}

The dynamic strength of composite materials is becoming a topic of increasing importance as the demand for energy efficient materials continues to rise. In reference [1] we have considered the basic problem of harmonic wave propagation in a composite consisting of a viscoelastic matrix reinforced by a doubly periodic array of cylindrical elastic fibers. As a further step towards a fuller understanding, in the present work we consider harmonic wave propagation in an elastic medium with a doubly periodic array of cylindrical pores.

Porosity can have a significant effect on the geometric dispersion of waves, and hence on dynamic strength, as indicated by the experiments of Okuko, Sve and Whittier [2]. A similar conclusion was reached by Nayfeh [3], who used a continuum theory of the Hegemier, Gurtman and Nayfeh type [4] to model propagation in a periodic array of perfectly bonded elastic laminates, one of which in turn contained a periodic array of cylindrical elastic inclusions (porosity being simulated by setting the values of the material constants to zero). Sve [5] also investigated a similar problem. He used a low frequency model to assess propagation normal to the laminates of a periodic, two-phase composite, one constituent having a random distribution of pores. Beltzer [6] presented a model for longitudinal wave propagation in which porosity was taken to be a Poisson stochastic process, the size, number and configuration of the pores being regarded as random parameters.

The work reported here is concerned with the problem of harmonic wave propagation in an elastic medium in which there is a periodic distribution of cylindrical pores of infinite length. As pointed out in reference [1], the geometrical complexity of such problems, even for a plane strain state such as considered here, renders them intractable analytically when linear, so-called "exact" theories are used. However as the work presented here and in reference [1] shows, and indeed this is in part the aim of the work, advances in finite element modeling and computational procedures prove to be adequate to meet the

† This work is part of a Ph.D. Dissertation in Applied Mechanics by the first author.

‡ Currently at Exxon Production Research Company, Houston, Texas 77001, U.S.A. 
challenge, even within the framework of modest budgets. Specifically, the field equations are transformed into integral expressions via Galerkin's method and then a finite element method leads to an algebraic eigenvalue problem, this latter being solved by modern matrix inversion procedures. Results on dispersion are given for several values of porosity. Also given are plots of amplitude and strain energy distributions within a typical half-cell.

As a check on the dynamic results, and as a contribution in its own right, equivalent elastic moduli are presented in the Appendix; these are obtained by using a static analysis similar to that given by Gaonkar [7]. This supplements the work of MacKenzie [8], who calculated the compressibility and shear modulus for a medium in which there was a random distribution of small spherical holes of different sizes and of Hashin [9] who gave bounds for the moduli of a medium in which there was a finite concentration of spherical elastic inclusions.

\section{FORMULATION OF THE PROBLEM}

The displacement equations of motion for a plane strain state in the absence of body forces may be written, on assuming harmonic time dependency (see reference [1]), as

$$
\begin{aligned}
& G\left(\partial^{2} u / \partial y^{2}+\partial^{2} v / \partial x \partial y\right)+\underline{\alpha} \partial^{2} u / \partial x^{2}+\underline{\beta} \partial^{2} v / \partial x \partial y+\rho \omega^{2} u=0 \\
& G\left(\partial^{2} u / \partial x \partial y+\partial^{2} v / \partial x^{2}\right)+\underline{\beta} \partial^{2} u / \partial x \partial y+\underline{\alpha} \partial^{2} v / \partial y^{2}+\rho \omega^{2} v=0
\end{aligned}
$$

where $G$ is the shear modulus, $\underline{\alpha}=K+\frac{4}{3} G, K$ being the bulk modulus, $\underline{\beta}=K-\frac{2}{3} G, \rho$ is the mass density and $\omega$ denotes radian frequency. The actual physical displacements $\bar{u}$ and $\bar{v}$ are given by

$$
\bar{u}(x, y, t)=u(x, y) \mathrm{e}^{\mathrm{i} \omega t}, \quad \bar{v}(x, y, t)=v(x, y) \mathrm{e}^{\mathrm{i} \omega t},
$$

so that $u$ and $v$ are in general complex.

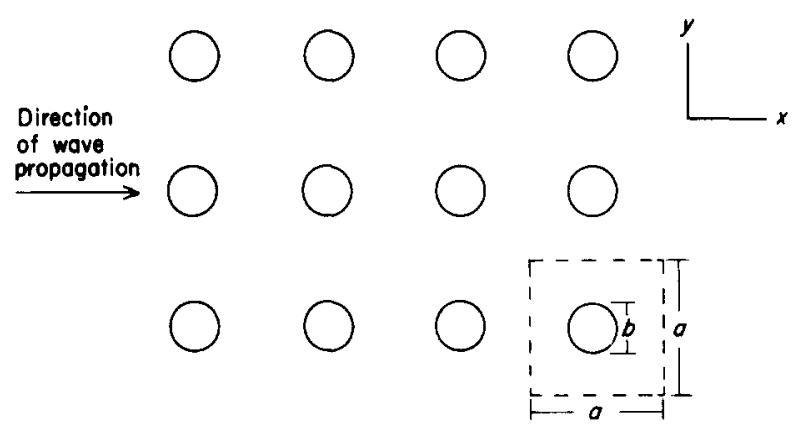

Figure 1. Doubly periodic configuration of the pores and a typical cell.

Symmetry dictates that only a typical half cell need be considered (see Figure 1). Then Floquet theory yields the following quasiperiodicity conditions, on taking the origin of co-ordinates at the pore center:

$$
\begin{gathered}
u\left(\frac{a}{2}, y\right)=\mathrm{e}^{\mathrm{i} q a} u\left(-\frac{a}{2}, y\right), \quad v\left(\frac{a}{2}, y\right)=\mathrm{e}^{\mathrm{i} q a} v\left(-\frac{a}{2}, y\right) \\
\frac{\partial}{\partial x} u\left(\frac{a}{2}, y\right)=\mathrm{e}^{\mathrm{i} q a} \frac{\partial}{\partial x} u\left(-\frac{a}{2}, y\right), \quad \frac{\partial}{\partial x} v\left(\frac{a}{2}, y\right)=\mathrm{e}^{\mathrm{i} q a} \frac{\partial}{\partial x} v\left(-\frac{a}{2}, y\right)
\end{gathered}
$$


where $q$ denotes the wavenumber of the harmonic wave. Since the wave is taken to be propagating parallel to the $x$-axis, symmetry requires that the normal displacements and shear stresses be zero on the half-cell boundaries at $y=0$ and $y=a / 2$ : i.e.,

$$
v(x, 0)=0=v\left(x, \frac{a}{2}\right), \quad \frac{\partial u}{\partial y}(x, 0)=0=\frac{\partial u}{\partial y}\left(x, \frac{a}{2}\right) .
$$

In addition, the pore boundaries are taken to be traction free so that the normal and shear stresses are zero there.

The above problem is not amenable to analytical techniques, and hence numerical methods must be employed; these will now be described.

\section{NUMERICAL PROCEDURES}

Galerkin's technique was employed in reference [1], and in view of the experience gained in that work, it was decided to use the same approach here. After using it and integration by parts, equations (1) and (2) become

$$
\begin{array}{r}
-\iint_{\Omega}\left\{G\left(\frac{\partial u}{\partial y}+\frac{\partial v}{\partial x}\right) \frac{\partial \phi}{\partial y}+\left(\underline{\alpha} \frac{\partial u}{\partial x}+\underline{\beta} \frac{\partial v}{\partial y}\right) \frac{\partial \phi}{\partial x}\right\} \mathrm{d} \Omega+\iint_{\Omega} \rho \omega^{2} u \phi \mathrm{d} \Omega \\
+\int_{\partial \Omega}\left\{G\left(\frac{\partial u}{\partial y}+\frac{\partial v}{\partial x}\right) \sin \bar{\alpha}+\left(\underline{\alpha} \frac{\partial u}{\partial x}+\underline{\beta} \frac{\partial v}{\partial y}\right) \cos \bar{\alpha}\right\} \phi \mathrm{d} s=0 \\
-\iint_{\Omega}\left\{G\left(\frac{\partial u}{\partial y}+\frac{\partial v}{\partial x}\right) \frac{\partial \phi}{\partial x}+\left(\underline{\beta} \frac{\partial u}{\partial x}+\underline{\alpha} \frac{\partial v}{\partial y}\right) \frac{\partial \phi}{\partial y}\right\} \mathrm{d} \Omega+\iint_{\Omega} \rho \omega^{2} v \phi \mathrm{d} \Omega \\
+\int_{\partial \Omega}\left\{G\left(\frac{\partial u}{\partial x}+\frac{\partial v}{\partial x}\right) \cos \bar{\alpha}+\left(\underline{\beta} \frac{\partial u}{\partial x}+\alpha \frac{\partial v}{\partial y}\right) \sin \bar{\alpha}\right\} \phi \mathrm{d} s=0 .
\end{array}
$$

Here $\Omega$ is the domain of interest, $\bar{\alpha}$ is the angle between the $x$-axis and the outward normal to the surface $\partial \Omega$ and equations (11) and (12) are to hold for all smooth functions $\phi$. Integration by parts was used to generate a form in which the boundary conditions are more readily handled.

A finite element method is now used. The domain $\Omega$ is divided into triangular subdomains (see Figure 2) in each of which $u$ and $v$ are approximated by

$$
u(x, y)=\sum_{j=1}^{3} u_{j} \phi_{j}(x, y), \quad v(x, y)=\sum_{j=1}^{3} v_{j} \phi_{j}(x, y),
$$

where the basis functions $\phi_{j}$ are taken to be linear functions over each subdomain and to satisfy

$$
\phi_{j}\left(x_{i}, y_{i}\right)=\delta_{i j} \text {, }
$$

where $\delta_{i j}$ is the Kronecker delta and $\left(x_{i}, y_{i}\right)$ are the co-ordinates of a node. Inserting equations (13) and (14) into equations (11) and (12) and performing the integrations leads to the matrix equation

$$
[K+S+M]\{u\}=0,
$$




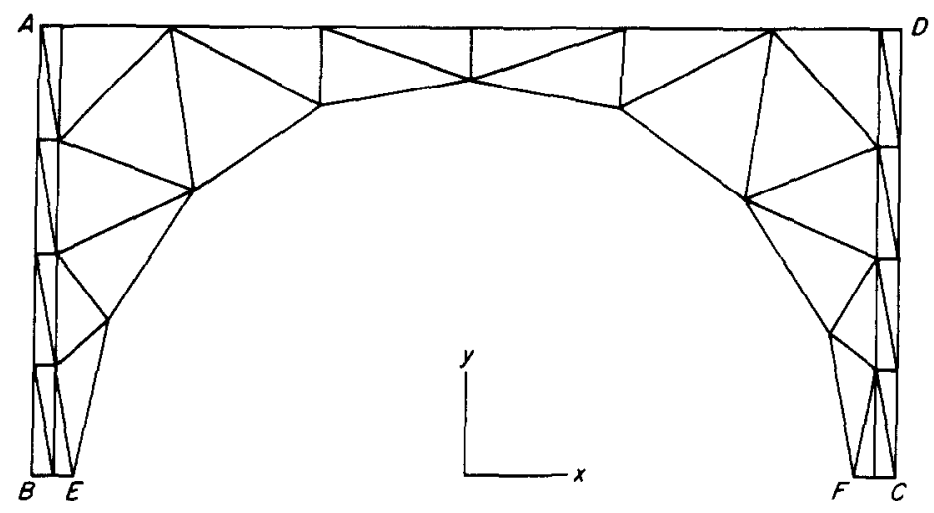

Figure 2. A typical half cell and its finite element mesh configuration.

where $K$ and $S$ are stiffness matrices, with $S$ involving the boundary conditions (9) and (10), $M$ is the mass matrix and $\{u\}^{\mathrm{T}}=\left(u_{1}, v_{1}, \ldots, u_{n}, v_{n}\right), n$ being the total number of nodes. The quasiperiodicity conditions (7) and (8) are handled as in reference [1] by adding, in the numerical work, a column outside the edge of the original discretization. Also as in reference [1], the algebraic eigenvalue problem stemming from equation (16) can be written as $A(\omega, q)\{\psi\}=0$, or, upon splitting $A$ and $\psi$ into real and imaginary parts, as

$$
N(\omega, q)\{\xi\}=0
$$

where

$$
N=\left[\begin{array}{cc}
A_{r} & -A_{i} \\
A_{i} & A_{r}
\end{array}\right], \quad\{\xi\}=\left\{\begin{array}{l}
\psi_{r} \\
\psi_{i}
\end{array}\right\} .
$$

The eigenvalues $\omega$ can be obtained from the function

$$
f(\omega)=\operatorname{det} N(\omega) \text {. }
$$

By using the so-called LU decomposition, $N(\omega)$ can be written as

$$
N(\omega)=\Pi L U,
$$

where $\Pi$ is a permutation matrix, $L$ is a lower triangular matrix with unit diagonal elements and $U$ is an upper triangular matrix. Then equation (19) can be written as

$$
f(\omega)= \pm \prod_{i=1}^{n} U_{i i}
$$

where the $U_{i i}$ are the diagonal elements of $U$. An iterative technique called the secant method is then used to determine the zeroes of $f(\omega)$. In this method, successive approximations to $\omega$ are determined from

$$
\omega_{k+2}=\left[\omega_{k+1} f\left(\omega_{k}\right)-\omega_{k} f\left(\omega_{k+1}\right)\right] /\left[f\left(\omega_{k}\right)-f\left(\omega_{k+1}\right)\right], \quad k=0,1,2, \ldots
$$

Equation (21) is used to monitor whether the eigenvalue is overshot or not.

\section{RESULTS}

The actual numerical procedure was as follows. First the values of $\omega$ for $q=0$ were found. Then, with attention focussed on a given mode, a non-zero value of $q$ was specified 
and, as an initial guess, $\omega$ was incremented by a small amount. In this way modes were swept out individually, proceeding from left to right.

A specific material was chosen to study the effects of the porosity $P$ (defined as the ratio of the void area to the area of the half cell without the void): namely, the aluminium alloy $2024-\mathrm{T} 4$, which has the properties $K=0.696 \times 10^{11} \mathrm{~N} / \mathrm{m}^{2}, G=0.276 \times 10^{11} \mathrm{~N} / \mathrm{m}^{2}, \rho=$ $0.277 \times 10^{4} \mathrm{~kg} / \mathrm{m}^{3}$.

The first seven modes are shown in Figures 3(a)-(d), where the dimensionless frequency $\bar{\omega}=\omega a \sqrt{\rho / K}$ is plotted against the dimensionless wavenumber $\bar{q}=q a$, for $P=0.636$, $0.385,0.196$ and 0.049 , respectively. Note that $\bar{q}$ is taken to vary between 0 and $\pi$, since, as shown by Nemat-Nasser [10], the dispersion relations in problems of this type are periodic with period $\pi$.

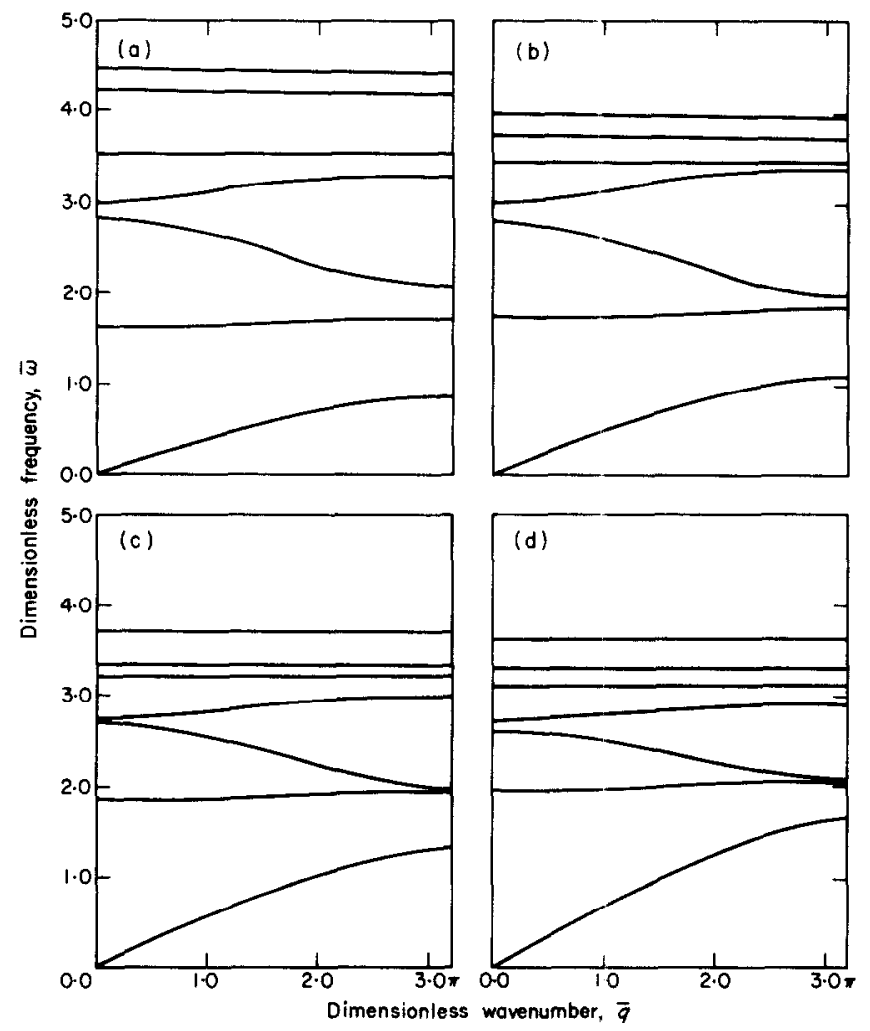

Figure 3. Dispersion relations. (a) Porosity $P=0.636$; (b) porosity $P=0.385$; (c) porosity $P=0.196$; (d) porosity $P=0 \cdot 049$.

An important point to note is the presence of the "no-pass bands": i.e., regions in which no real wavenumbers can be found for certain frequencies. Such bands have also been discovered in related works (see reference [10]). Also, the following trend may be inferred as $P$ goes to zero: the first mode tends to become straight and the remaining curves combine to form a horizontal line, this latter representing a non-propagating event. These observations are consistent with the fact that in an infinite medium there is only one wave speed for longitudinal propagation.

The largest value of the group velocity $d \bar{\omega} / \mathrm{d} \bar{q}$ occurs in the first mode at zero values of frequency and wavenumber. This observation could lend weight to approximate theories based on large wavenumbers and low frequencies. This group velocity (normalized by its 


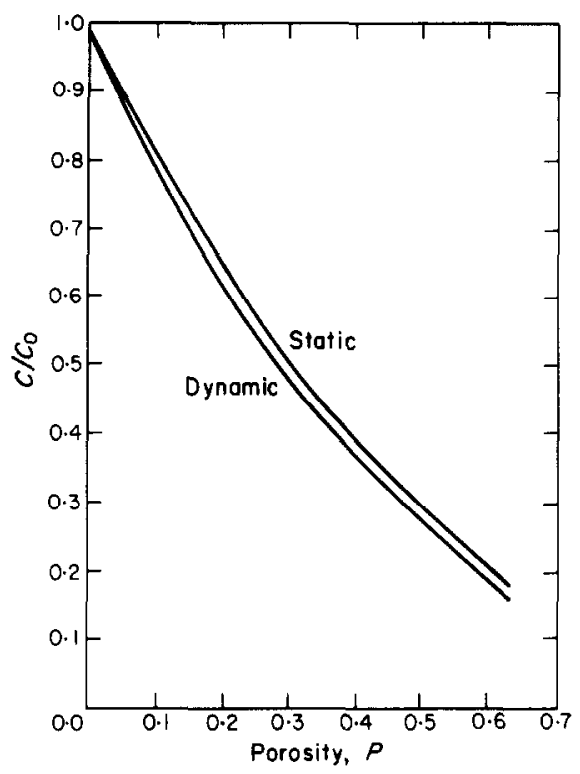

Figure 4. Comparison between the static and dynamic values of the normalized, infinite wavelength, lowest mode group velocity $C / C_{0}$ as a function of the porosity $P$.

zero porosity value) is shown as a function of porosity $P$ in Figure 4 . Shown there also is the same quantity obtained from an "equivalent moduli" approach presented in the Appendix. As can be seen, the comparison is very good with, in both cases, the group velocity decreasing uniformly with increasing values of $P$. Note also in Figures 3(a)-(d) that the group velocities for the higher modes are zero at $\bar{q}=0$ and $\pi$. This has been experimentally verified in other cases (see reference [11]).

Some studies on amplitude and energy distributions were also undertaken. The physical quantities $\bar{u}$ and $\bar{v}$ can be expressed in the form

$$
\bar{u}=A_{u} \cos \left(\omega t+\phi_{u}\right), \quad \bar{v}=A_{v} \cos \left(\omega t+\phi_{v}\right),
$$

where $A_{u}$ and $A_{v}$ are amplitudes, and $\phi_{u}$ and $\phi_{v}$ are phase angles. Figures 5(a) and (b) show dimensionless first mode amplitudes $A_{u}$ and $A_{v}$ as functions of $x / a$ for the wavenumber $\bar{q}=\pi$ (this implies that the wavelength $\lambda=2 a$ ), elevation $y=0.325 a$ and porosity $P=0 \cdot 196$. Note that the scale of the ordinate in Figure $5(\mathrm{~b})$ is $1 / 20$ of that in Figure 5(a), which implies that the displacement in the $y$-direction is much smaller than that in the $x$-direction. This is due to the boundary conditions confining the motion in the $y$-direction along the upper and lower edges.

Another quantity of interest is the strain energy. For a plane strain state, the strain energy $W_{1}$ of a single element can be written in terms of the strains $\varepsilon_{x x}, \varepsilon_{y y}$ and $\varepsilon_{x y}$ as follows:

$$
W_{1}=\frac{1}{2} \iint_{\Omega_{1}}\{\varepsilon\}^{\mathrm{T}}[H]\{\varepsilon\} \mathrm{d} \Omega_{1}
$$

where

$$
\{\varepsilon\}=\left\{\begin{array}{c}
\varepsilon_{x x} \\
\varepsilon_{y y} \\
2 \varepsilon_{x y}
\end{array}\right\}, \quad[H]=\frac{E}{1-\nu^{2}}\left[\begin{array}{ccc}
1, & \nu, & 0 \\
\nu, & 1, & 0 \\
0, & 0, & (1-\nu / 2)
\end{array}\right]
$$




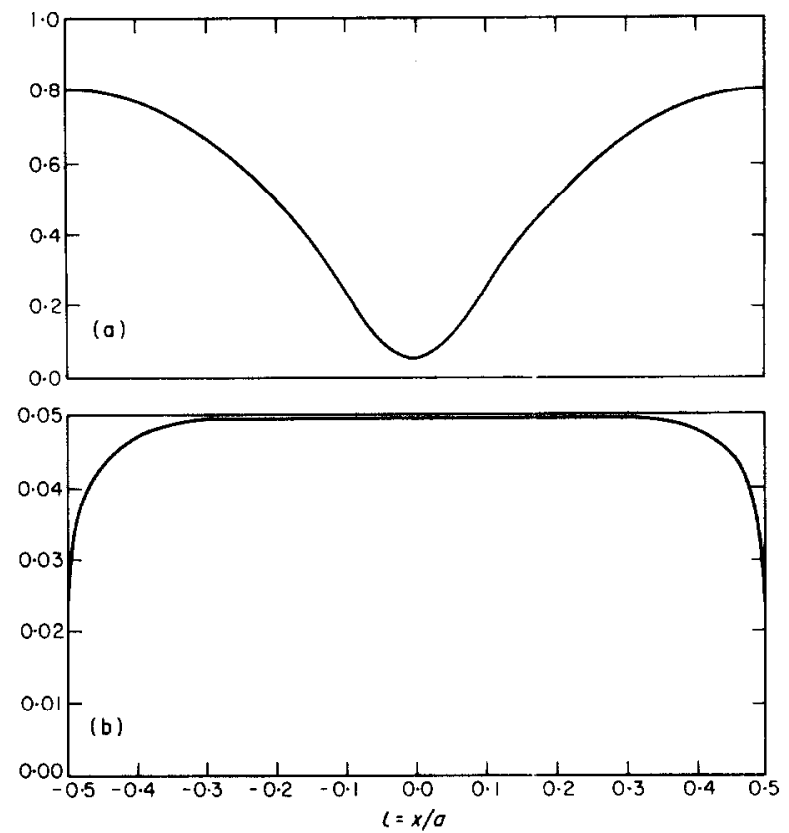

Figure 5. Dimensionless first mode amplitude (a) $\boldsymbol{A}_{u}$ and (b) $\boldsymbol{A}_{v}$ as a function of $x / a . \bar{q}=\pi, y=0.325 a$, $P=\mathbf{0} \cdot 196$.

By using the displacement fields given by equatiuns (13), (14) and (15) and the standard strain displacement relations, $\{\varepsilon\}$ and hence $W_{1}$ can be calculated. Then the strain energy distribution in a typical half cell can be obtained, with the value for each triangular element taken to be localized at its centroid. Figure 6(a) shows contours of constant, dimensionless strain energy in the first mode for $\bar{q}=\pi$ and the porosity $P=0 \cdot 049$. Note that a symmetric
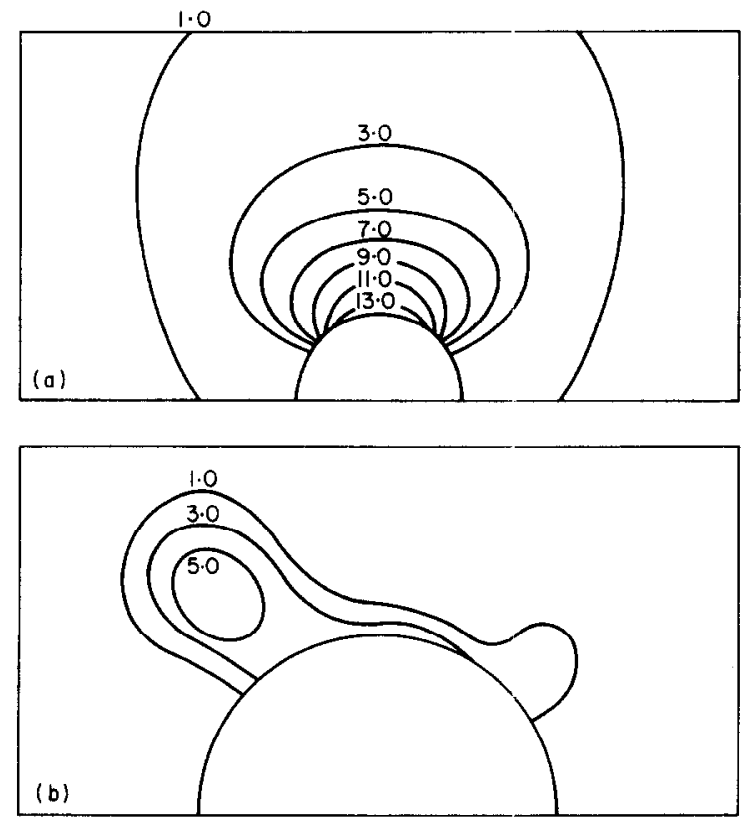

Figure 6. Dimensionless strain energy distribution in the first mode. (a) $\bar{q}=\pi, P=0.049$; (b) $\bar{q}=2.4$, $P=0 \cdot 196$. 
pattern is obtained since the wavelength $\lambda=2 a$. Note further that the strain energy has its peak values in the vicinity of the top of the pore, showing that dynamic stress concentration has occurred. Figure 6(b) gives first mode contours for $P=0.196$ and $\bar{q}=2.4$, which corresponds to $\lambda=2.62 a$. The pattern is now unsymmetric since $\lambda$ is not an integer multiple of $a$. It is interesting to note that the peak values of the strain energy are not in the immediate vicinity of the void, but instead occur near the location of the peak amplitude of the incoming wave.

\section{REFERENCES}

1. T.-C. MA, R. A. SCOTT and W. H. YANG 1980 Journal of Sound and Vibration 69, 257-264. Harmonic wave propagation in an infinite viscoelastic medium with a periodic array of cylindrical elastic fibers.

2. S. OKuko, C. SVE and J. S. WhITTIER 1972 Aerospace Corporation Report No. TOR-0712 (2816-75) - 1. Dispersion of an elastic step pulse in 3DQP-effect of porosity.

3. A. H. NA YFEH 1975 International Joumal of Solids and Structures 11, 937-946. The influence of porosity on the elastic response of homogeneous and structural composite media.

4. G. A. HEGEMIER, G. A. GURTMAN and A. H. NAYFEH 1973 International Journal of Solids and Structures 9, 395-414. A continuum mixture theory of wave propagation in laminated and fiber-reinforced composites.

5. C. SVE 1973 International Journal of Solids and Structures 9, 937-950. Elastic wave propagation in a porous laminated composite.

6. A. BELTZER 1978 Journal of Sound and Vibration 58, 251-256. The influence of random porosity on elastic wave propagation.

7. G. H. GAONKAR 1969 Journal of Applied Mechanics 36, 134-138. Uniaxial loading in an elastic continuum with a doubly periodic array of material discontinuities.

8. J. K. MACKENZIE 1950 Proceedings of the Physical Society B63, 2-11. The elastic constants of a solid containing spherical holes.

9. Z. HASHIN 1962 Journal of Applied Mechanics 29, 143-150. The elastic moduli of heterogeneous materials.

10. S. NemAT-NASSER 1976 Mechanics Today (Volume 3). New York: Pergamon Press.

11. G. H. GolUB, L. JENNING and W. H. YANG 1975 Journal of Computational Physics 17, 349-357. Waves in periodicically structured media.

12. S. LeIBORICH 1974 Nonlinear Waves. London: Cornell University Press.

\section{APPENDIX: EQUIVALENT MODULI FOR A POROUS MEDIUM}

In this appendix, equivalent moduli (and density) for a porous medium will be calculated by using Gaonkar's [7] static method. This is done partly as a check on some of the results obtained by using the dynamic analysis, but also it is felt to have some value in its own right.

To begin, the boundary conditions are taken to be (see Figure 2)

$$
\begin{gathered}
u(-a / 2, y)=C_{1} \text { on } A B, \quad v(x, a / 2)=C_{2} \text { on } A D, \\
u(a / 2, y)=0 \text { on } C D, \quad v(x, 0)=0 \text { on } B E, F C,
\end{gathered}
$$

where $C_{1}$ and $C_{2}$ are constants, being the relative displacements between $A B$ and $C D$, and $A D$ and $B E$ (or $F C$ ), respectively. In addition, the curve $E F$ is taken to be traction free.

By using a finite element method (here actually one based on Rayleigh-Ritz techniques was used), the equilibrium equations can be written in the matrix form

$$
\left[\boldsymbol{K}_{\boldsymbol{s}}\right]\{u\}=\{f\},
$$

where $\{f\}$ is a $2 n \times 1$ nodal force vector, $n$ being the number of nodes. Equation (A5) together with the boundary conditions can be solved by using Gaussian elimination techniques, and by this procedure the unknown nodal forces are determined. Then, 
average stresses are defined by (for unit thickness in the $y$-direction)

$$
\bar{\sigma}_{x x}=\frac{2}{a} \sum_{i} f_{x}^{i}, \quad \bar{\sigma}_{y y}=\frac{1}{a} \sum_{j} f_{y}^{i}, \quad \bar{\sigma}_{x y}=\frac{2}{a} \sum_{i} f_{y}^{i}
$$

where $f_{x}^{i}$ and $f_{y}^{i}$ are the $x$ - and $y$-nodal force components at the ith node on the side $A B$ and $f_{y}^{j}$ is the $y$-nodal force component at the $j$ th node on the side $A D$.

By analogy with the stress-strain relations for a plane strain state, equivalent moduli for the porous medium are defined by

$$
\begin{gathered}
\bar{\sigma}_{x x}=[\bar{E} /(1+\bar{\nu})(1-2 \bar{\nu})]\left[(1-\bar{\nu}) \bar{\epsilon}_{x x}+\bar{\nu} \bar{\varepsilon}_{y y}\right], \\
\bar{\sigma}_{y y}=[\bar{E} /(1+\bar{\nu})(1-2 \bar{\nu})]\left[\bar{\nu} \bar{\varepsilon}_{x x}+(1-\bar{\nu}) \bar{\varepsilon}_{y y}\right], \\
\bar{\sigma}_{x y}=[\bar{E} /(1+\bar{\nu})] \bar{\varepsilon}_{x y},
\end{gathered}
$$

in which, $\bar{E}$ and $\bar{\nu}$ are equivalent moduli, $\bar{\sigma}_{x x}, \bar{\sigma}_{y y}$ and $\bar{\sigma}_{x y}$ are as given in equations (A6), (A7) and (A8) and $\bar{\varepsilon}_{x x}, \bar{\varepsilon}_{y y}$ and $\bar{\varepsilon}_{x y}$ are average normal and shear strains which are approximated by

$$
\bar{\varepsilon}_{x x}=C_{1} / a, \quad \bar{\varepsilon}_{y y}=2 C_{2} / a, \quad \bar{\varepsilon}_{x y}=0 .
$$

In equations (A9), (A10) and (A11), $\bar{\sigma}_{x x}, \bar{\sigma}_{y y}, \bar{\sigma}_{x y}, \bar{\varepsilon}_{x x}, \bar{\varepsilon}_{y y}$ and $\bar{\varepsilon}_{x y}$ are known quantities so that $\bar{E}$ and $\bar{\nu}$ can be obtained.

The corresponding equivalent density $\bar{\rho}$ is defined by $\bar{\rho}=(1-P) \bar{\rho}_{0}$, where $P$ is the porosity and $\bar{\rho}_{0}$ is the density when $P$ is equal to zero.
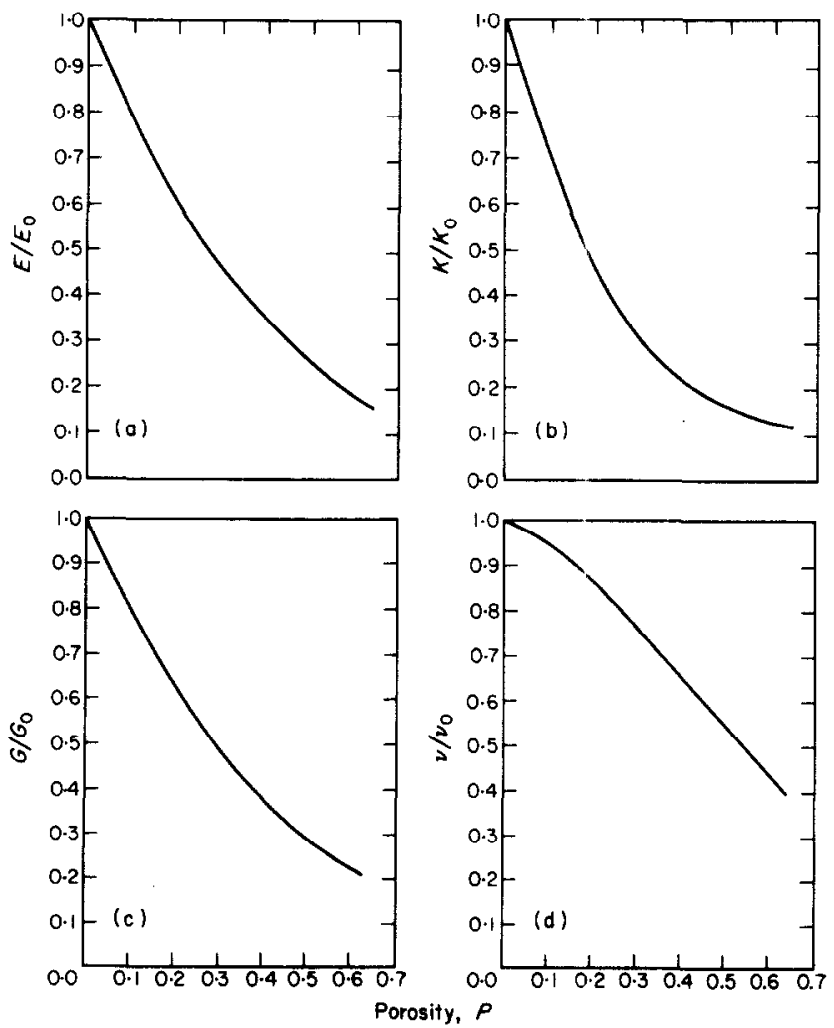

Figure A1. Variation with the porosity $P$ of the normalized equivalent (a) Young's modulus $E / E_{0}$, (b) bulk modulus $K / K_{0}$, (c) shear modulus $G / G_{0}$ and (d) Poisson's ratio $\nu / \nu_{0}$. 
The boundary conditions in equations (9) and (10) are different from those in this appendix. The former have zero displacements in the $y$-direction on the upper and lower edges of the half cell and the latter have not. Equivalence can be obtained by modifying $\bar{E}$ in this appendix (the so-called "laterally unconstrained case") to $\bar{E}^{\prime}$ (the so-called "laterally constrained case") by (see reference [12])

$$
\bar{E}^{\prime}=\bar{E}(1-\bar{\nu}) /(1+\bar{\nu})(1-2 \bar{\nu}) \text {. }
$$

The ratio $C=\sqrt{\bar{E}^{\prime} / \bar{\rho}}$ is the infinite wavelength group velocity of waves propagating in the lowest mode. A comparison between the normalized value of it and the dynamic results is given in Figure 4, which, to repeat, shows excellent agreement.

As items of interest and value in their own right, Figures $A 1(a)-(d)$ show the variations of normalized equivalent values of Young's modulus, the bulk modulus, the shear modulus and the Poisson's ratio, with the porosity $P$. The normalizing factors are the zero porosity values. 